\author{
Fumiko Matsuzawa $\cdot$ Sei-ichi Aikawa $\cdot$ Hitoshi Sakuraba \\ Hoang Thi Ngoc Lan · Akemi Tanaka Kousaku Ohno \\ Yuko Sugimoto $\cdot$ Haruaki Ninomiya · Hirofumi Doi
}

\title{
Structural basis of the GM2 gangliosidosis B variant
}

Received: 17 July 2003 / Accepted: 6 September 2003 / Published online: 24 October 2003

(C) The Japan Society of Human Genetics and Springer-Verlag 2003

\begin{abstract}
To study the structural basis of the GM2 gangliosidosis B variant, we constructed the threedimensional structures of the human $\beta$-hexosaminidase $\alpha$-subunit and the heterodimer of the $\alpha$ - and $\beta$-subunits, Hex A, by homology modeling. The $\alpha$-subunit is composed of two domains, domains I and II. Nine mutant models due to specific missense mutations were constructed as well and compared with the wild type to determine structural defects. These nine mutations were divided into five groups according to structural defects. $\mathrm{R} 178 \mathrm{H}$ is deduced to affect the active site directly, because R178 is important for binding to the substrate.
\end{abstract}

F. Matsuzawa $\cdot$ S. Aikawa $\cdot$ H. Doi $(\bowtie)$

Celestar Lexico-Sciences, Inc.,

MTG D-17, 1-3 Nakase,

Mihama-ku, Chiba 261-8501, Japan

E-mail: doi@cl-sciences.co.jp

Tel.: + 81-43-2745801

Fax: + 81-43-2745817

H. Sakuraba

Department of Clinical Genetics,

The Tokyo Metropolitan Institute of Medical Science,

Tokyo Metropolitan Organization for Medical Research,

3-18-22 Honkomagome, Bunkyo-ku,

Tokyo 113-8613, Japan

H. T. N. Lan · A. Tanaka

Department of Pediatrics,

Osaka City University Graduate School of Medicine,

1-4-3 Asahi-machi, Abeno-ku,

Osaka 545-8585, Japan

K. Ohno

Department of Pediatric Neurology,

School of Life Sciences,

Tottori University Faculty of Medicine,

86 Nishi-machi,

Yonago 683-0826, Japan

Y. Sugimoto $\cdot$ H. Ninomiya

Department of Neurobiology,

School of Life Sciences,

Tottori University Faculty of Medicine,

86 Nishi-machi,

Yonago 683-0826, Japan
C458Y and W420C are predicted to cause drastic structural changes in the barrel structure carrying the active site pocket. $\mathrm{R} 504 \mathrm{C} / \mathrm{H}$ is deduced to introduce a disruption of an essential binding with D494 in the $\beta$ subunit for dimerization. $\mathrm{R} 499 \mathrm{C} / \mathrm{H}$, located in an extrahelix, is deduced to disrupt hydrogen bonds with domain I and the barrel. R170W and L484P are deduced to affect the interface between domains I and II, causing destabilization. The structural defects reflect the biochemical abnormalities of the disease.

Keywords GM2 gangliosidosis - Tay-Sachs disease · $\beta$-hexosaminidase $\cdot$ Homology modeling $\cdot$ GM2 ganglioside

\section{Introduction}

There are two major $\beta$-hexosaminidase (Hex, EC 3. 2. 1. 52) isozymes in humans, Hex $\mathrm{A}(\alpha \beta$, a heterodimer of the $\alpha$ - and $\beta$-subunits), and Hex B ( $\beta \beta$, a homodimer of the $\beta$-subunits), and a very small amount of an unstable isozyme, Hex S $(\alpha \alpha$, a homodimer of the $\alpha$-subunits). All three Hex isozymes can catalyze the hydrolysis of terminal $\beta$-1, 4-linked $N$-acetylglucosamine (GlcNAc) and $N$-acetylgalactosamine (GalNAc) residues at the nonreducing ends of oligosaccharides and their conjugates. However, only Hex A and Hex S can cleave terminal nonreducing $N$-acetylglucosamine6-sulfate (GlcNAc-6-S) residues in keratan sulfate, and Hex A is essential for cleavage of the GalNAc residue from the GM2 ganglioside.

The GM2 gangliosidosis B variant (McKusick 272800 ) is an autosomal recessive genetic disorder caused by mutations of HEXA, which codes for the Hex $\alpha$-subunit, on chromosome $15 \mathrm{q} 23-24$. The resulting deficiency of Hex A $(\alpha \beta)$ causes pathological accumulation of the GM2 ganglioside in the central nervous system, which results in neurological disorders (Gravel et al. 2001). The disease exhibits a spectrum of clinical phenotypes ranging from the severe infantile form 
(classical Tay-Sachs disease) to the milder late onset form (juvenile type and adult type). Efforts have been made to identify mutations of the HEXA gene, and many disease-causing mutations, including amino acid substitutions, have been found (Gravel et al. 2001). However, structural studies on the defective $\alpha$-subunit have not been performed yet, although this is very important for clarifying the pathogenesis of the disease.

The crystal structures of chitobiose from Serratia marcescens (Tews et al. 1996), which is a member in the same family 20 glycosyl hydrolase as Hex; and Hex from Streptomyces plicatus (Mark et al. 2001), were refined, and the data were deposited in the Protein Data Bank (PDB) (Berman et al. 2000). We then modeled the human Hex $\beta$-subunit by means of homology modeling using this information and characterized the molecular defect of the Hex $\beta$-subunit causing the GM2 gangliosidosis 0 variant (Sandhoff disease and variants) (Sakuraba et al. 2002). Recently, the crystal structure of human Hex B was determined by Mark et al. (2003) and followed by Maier et al. (2003). The deduced locations of the mutations on our model were consistent with those of the determined structure, which indicated that our model was reliable and effective in analyzing the molecular defect causing the disease.

In this study, we further constructed a structural model of the human Hex $\alpha$-subunit and studied the structural defects caused by amino acid substitutions responsible for the GM2 gangliosidosis B variant.

\section{Materials and methods}

Clinical summary and cell culture

Cultured skin fibroblasts from ten independent patients with the GM2 gangliosidosis B variant and a normal control subject were established and maintained in our laboratory. Patient data, including their ethnic origins, phenotypes and genotypes, are summarized in Table 1 . Case 1 is a Puerto Rican and the others (cases 2-10) are of Japanese origin. Three patients (cases 1, 6, and 7) presented a clinical profile of the juvenile type of the disease, and the others exhibited one of classical Tay-Sachs disease. All patients except for cases 1,8 , and 10 each have a specific missense mutation (W420C, C458Y, R504C, R504H, R499C, R499H, or L484P) on one allele and a common splicing mutation (IVS5, -1G $\rightarrow$ T) in Japanese Tay-Sachs patients (Tanaka et al. 1993) on the other allele. Case 1 is heterozygous for R178H and 1278insTATC, which is one of the common mutations in Ashikenazi Jewish Tay-Sachs patients (Myerowitz and Costigan 1988). Cases 8 and 10 are homozygous for R170W and the common Japanese splicing mutation, respectively. The cells were cultured in Eagle's minimal essential medium containing $10 \%$ fetal bovine serum at $37^{\circ} \mathrm{C}$ under $5 \% \mathrm{CO}_{2}$.

\section{Enzyme assay and kinetic study}

Hex A activity was determined in total homogenates of cultured fibroblasts using 4-methylumbelliferyl GlcNAc-6-S (HSC Research Development Co., Toronto, Canada) as the substrate (Suzuki 1987). The amount of protein was determined with the Bio-Rad dye-binding assay (Bio-Rad Laboratories, Richmond, CA, USA) with bovine serum albumin as the standard. The apparent $\mathrm{Km}$ values were determined with fibroblast extracts from case 1 and a normal control subject as the enzyme sources by means of a Lineweaver-Burke double reciprocal plot.

Structural modeling of human wild-type and mutant Hex $\alpha$-subunits

Structural models of the human wild-type Hex $\alpha$-subunit, its mutants (R170W, R178H, W420C, C458Y, L484P, R499C/H and $\mathrm{R} 504 \mathrm{C} / \mathrm{H})$ and Hex A were built using molecular modeling software, SYBYL/COMPOSER and BIOPOLYMER (TRIPOS, Inc., Mountain View, CA, USA), based on the crystal structure of the human Hex B (PDB ID; 1NOU) as template. The backbone of the human wild type Hex $\alpha$-subunit corresponding to each structurally conserved region was built by fitting a fragment of the template,

Table 1 Phenotypes and genotypes of the patients with the GM2 gangliosidosis B variant

\begin{tabular}{|c|c|c|c|c|}
\hline Case no. & Origin & Pheno type & Hex $A$ activity ${ }^{a}$ & Geno type \\
\hline $1^{\mathrm{b}}$ & $\begin{array}{l}\text { Puerto } \\
\text { Rican }\end{array}$ & Juvenile & 0.02 & $\mathrm{R} 178 \mathrm{H}^{\mathrm{c}} / 1278$ insTATC ${ }^{\mathrm{d}}$ \\
\hline $2^{\mathrm{e}}$ & Japanese & Infantile & $<0.01$ & $\mathrm{~W} 420 \mathrm{C}^{\mathrm{f}} / \mathrm{IVS} 5,-1 \mathrm{G} \rightarrow \mathrm{T}^{\mathrm{g}}$ \\
\hline $3^{\mathrm{h}}$ & Japanese & Infantile & $<0.01$ & $\mathrm{C} 458 \mathrm{Y}^{\mathrm{h}} / \mathrm{IVS} 5,-1 \mathrm{G} \rightarrow \mathrm{T}$ \\
\hline $4^{\mathrm{h}}$ & Japanese & Infantile & 0.01 & $\mathrm{R}^{2} 04 \mathrm{C}^{\mathrm{i}} / \mathrm{IVS} 5,-1 \mathrm{G} \rightarrow \mathrm{T}$ \\
\hline $5^{\mathrm{h}}$ & Japanese & Infantile & $<0.01$ & $\mathrm{R} 504 \mathrm{H}^{\mathrm{j}}+\mathrm{S} 302 \mathrm{G}^{\mathrm{k}} / \mathrm{IVS} 5,-1 \mathrm{G} \rightarrow \mathrm{T}$ \\
\hline $6^{\mathrm{e}}$ & Japanese & Juvenile & 0.02 & $\mathrm{R}_{4} 9 \mathrm{C}^{\mathrm{l}} / \mathrm{IVS} 5,-1 \mathrm{G} \rightarrow \mathrm{T}$ \\
\hline $7^{\mathrm{e}}$ & Japanese & Juvenile & 0.03 & $\mathrm{R} 49 \mathrm{H}^{\mathrm{m}} / \mathrm{IVS} 5,-1 \mathrm{G} \rightarrow \mathrm{T}$ \\
\hline $8^{\mathrm{n}}$ & Japanese & Infantile & $<0.01$ & $\mathrm{R} 170 \mathrm{~W}^{\mathrm{o}} / \mathrm{R} 170 \mathrm{~W}$ \\
\hline $9^{\mathrm{h}}$ & Japanese & Infantile & $<0.01$ & $\mathrm{~L} 484 \mathrm{P}^{\mathrm{h}} / \mathrm{IVS} 5,-1 \mathrm{G} \rightarrow \mathrm{T}$ \\
\hline $10^{\mathrm{p}}$ & Japanese & Infantile & $<0.01$ & IVS5,-1G $\rightarrow$ T/IVS5,-1G $\rightarrow$ T \\
\hline 11 & Japanese & Normal & 0.62 & Normal/normal \\
\hline
\end{tabular}

${ }^{\mathrm{a}}$ Enzyme unit: $\mu \mathrm{mol} / \mathrm{mg} \operatorname{prot} / \mathrm{h}$;

${ }^{\mathrm{b}}$ Tanaka et al. (1988), Tanaka et al. (1990);

${ }^{\mathrm{c}}$ Tanaka et al. (1990), Ohno et al. (1988), Kytzia et al. (1983), dos

Santos et al. (1991), Tanaka et al. (1988);

${ }^{\mathrm{d}}$ Myerowitz and Costigan (1988), Nishimoto et al. (1991), Paw and

Neufeld (1988);

'e Submitted by Tanaka et al., Tanaka et al. (1990);

${ }^{\mathrm{g}}$ Tanaka et al. (1993);

hanaka et al. (1994),
${ }^{\mathrm{i}}$ Paw et al. (1991), Akli (1991);

${ }^{j}$ Paw et al. (1990), Akli et al. (1993), Boustany et al. (1991);

${ }^{\mathrm{k}} \mathrm{S} 302 \mathrm{G}$ is thought to be a polymorphism;

${ }^{1}$ Mules et al. (1992);

${ }^{m}$ Paw et al. (1990);

${ }^{\mathrm{n}}$ Tanaka et al. (1999);

${ }^{\circ}$ Fernandes et al. (1992);

p Tanaka et al. (1993) 
and the side chain conformations were determined by means of the rule-based procedure (Sutcliffe 1987). Loop regions were constructed with fragments selected from the protein substructure database (Jones and Thirup 1986; Claessens et al. 1989). The energy minimization procedure was used to optimize the conformations and side chain rotamers. The mutant models were built in the same way as for the wild type, but based on the primary structures with the amino acid replacements. To evaluate the influence of each replacement on the model structure, the mutant model was superimposed with the wild type model based on the $\mathrm{C} \alpha$ atoms by a least-square-mean fitting method (Kabsh 1976, 1978). We defined that the structure was influenced by the amino acid replacement when the position of an atom of the mutant differed from that in the case of the wild type by more than the total root-mean-square distance value.

\section{Results}

Hex A activity in cultured fibroblasts

In cases $2-5$ and $8-10$ with the phenotype of classic TaySachs disease, Hex A activity was less than $2 \%$ of the normal control level. In cases 1,6 , and 7 with the juvenile type of the disease, Hex A activity was about 3$5 \%$ of the control level (Table 1). Because both these mutations, including an insertion of 4 base-pairs in exon 11 (1278insTATC) and the splicing mutation (IVS5, $-1 \mathrm{G} \rightarrow \mathrm{T}$ ), could not express any catalytic activity (Nishimoto et al. 1991 and the results of the enzyme assay in case 10), the residual Hex A activity in cases 1, 6 , and 7 is thought to have resulted from the allele carrying the specific missense mutation (R178H, R499C or $\mathrm{R} 499 \mathrm{H})$.

Structural modeling of human wild-type Hex $\alpha$-subunit

A structural model of the human Hex $\alpha$-subunit was built using the crystallographic data for the human Hex $\beta$-subunit as template. According to the sequence alignment, we built a model of the human Hex $\alpha$-subunit composed of the 502 amino acids from residues 23 to 524. The amino acid identity between $\alpha$ - and $\beta$-subunits was $54.7 \%$. The modeled structure of the Hex $\alpha$-subunit is shown in Fig. 1. It comprises two domains (I and II). Domain I (residues 23-165) has an $\alpha / \beta$ topology, and domain II (residues 166-524) is folded into a $(\beta / \alpha)$ 8 -barrel with the active site pocket at the C-termini of $\beta$-strands. An extra-helix that follows the eighth helix of the $(\beta / \alpha)_{8}$-barrel is located between domain I and the barrel structure.

Predicted structural changes in the human Hex $\alpha$-subunit due to amino acid substitutions causing the GM2 gangliosidosis B variant

The positions of mutations were mapped in the modeled three-dimensional structure of the wild-type Hex $\alpha$ subunit (Fig. 1) and the heterodimer model (Fig. 2). All substituted residues are conserved between human $\alpha$ and $\beta$-subunits. To determine the influence of the mutations, we constructed mutant models and compared them with the wild type. As a result, we divided the nine mutations into five groups according to the structural defects (Table 2).

\section{Group $1(\mathrm{R} 178 \mathrm{H})$}

The R178 residue is located at the loop following the first $\beta$-strand of the $(\beta / \alpha)_{8}$-barrel and is close to the active site (Fig. 1). Substitution of R178 to H caused conformational changes in the residues (D207, D208, H262, E323, N423, and E462), which are responsible for the enzymatic activity (Fig. 3a). A structural change was observed only inside the active site pocket.

In case 1 carrying this mutation, the apparent $\mathrm{Km}$ value was estimated to be $1.5 \mathrm{mM}$, which is about 30 times higher than that in the control subject $(0.05 \mathrm{mM})$. These data suggest that a structural change occurred in (or near) the active site of the $\alpha$-subunit (Figs. 1 and 3a).

\section{Group 2 (W420C and C458Y)}

The mutations C458Y and W420C in this group were located on the $\beta$-strands of the barrel enclosing the active site pocket (Fig. 1). The C458 residue is located on the eighth $\beta$-strand, and the side chain points inside the active site pocket. Substitution of C458 to Y with a long and bulky side chain caused steric clashes with the surrounding residues, thereby causing a drastic structural change in the whole active site pocket (Fig. 3b). The structural change caused by $\mathrm{C} 458 \mathrm{Y}$ is larger than that by R178H (Fig. 3).

The $\mathrm{W} 420$ residue is in the seventh $\beta$-strand. The side chain is buried inside the barrel and involved in hydrophobic interactions with L416, F434, V437 and V480 interior of the barrel, which are thought to stabilize the barrel structure enclosing the active site pocket. Substitution of W420 to C causes loss of the hydrophobic interactions, thus causing a conformational change around the active site pocket (data not shown).

\section{Group 3 (R504C and R504H)}

R504 is located in the extra-helix in domain II (Fig. 1). This residue points outside of the extra-helix and is exposed in the monomer. As shown in Fig. 2, R504 of the $\alpha$-subunit directly binds to D494 of the $\beta$-subunit in the $\alpha \beta$ heterodimer model. Substitution of R504 to $\mathrm{C}$ or $\mathrm{H}$ causes a disruption of the binding between the $\alpha$ - and $\beta$-subunits and a moderate conformational change on the surface near the residue (Fig. 4a). 
Fig. 1A, B Three-dimensional structure of the human $\beta$-hexosaminidase $\alpha$-subunit. A Side view of the human $\beta$-fhexosaminidase $\alpha$-subunit. B Front view of the protein. The secondary structures are shown as tube and ribbon drawings; $\alpha$-helix (magenta), $\beta$-sheet (yellow), and coil (cyan). Roman numerals indicate domains. Residues forming disulfide bonds (C58-C104, C277-C328, and C505-C522) and residues involved in the catalytic triad (D207, H262 and E323) are presented as ball-andsticks models. Residue positions involved in amino acid substitutions are presented as space-filling models. Domain I has an $\alpha / \beta$ topology. Domain II has a $(\beta / \alpha)_{8}$-barrel structure
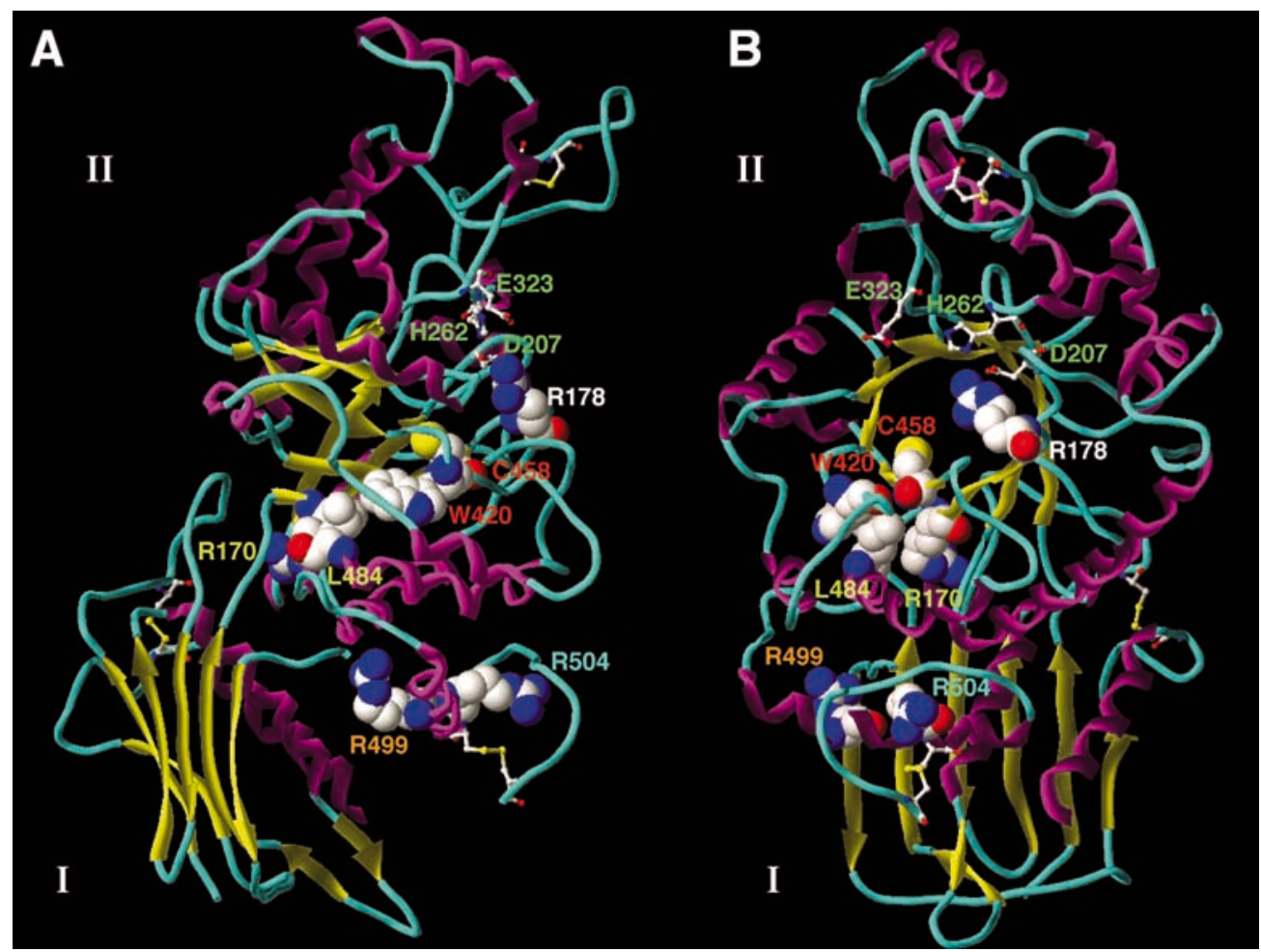

Fig. 2 Three-dimensional structure of the human $\beta$ hexosaminidase heterodimer of the $\alpha$ - and $\beta$-subunits. The $\alpha$ - and $\beta$-subunits are shown in cyan and yellow, respectively. Roman numerals indicate domains. Residue positions involved in amino acid substitutions in the $\alpha$-subunit and the residue of D494 in the $\beta$-subunit, which binds to R504 in the $\alpha$-subunit, are presented as space-filling models

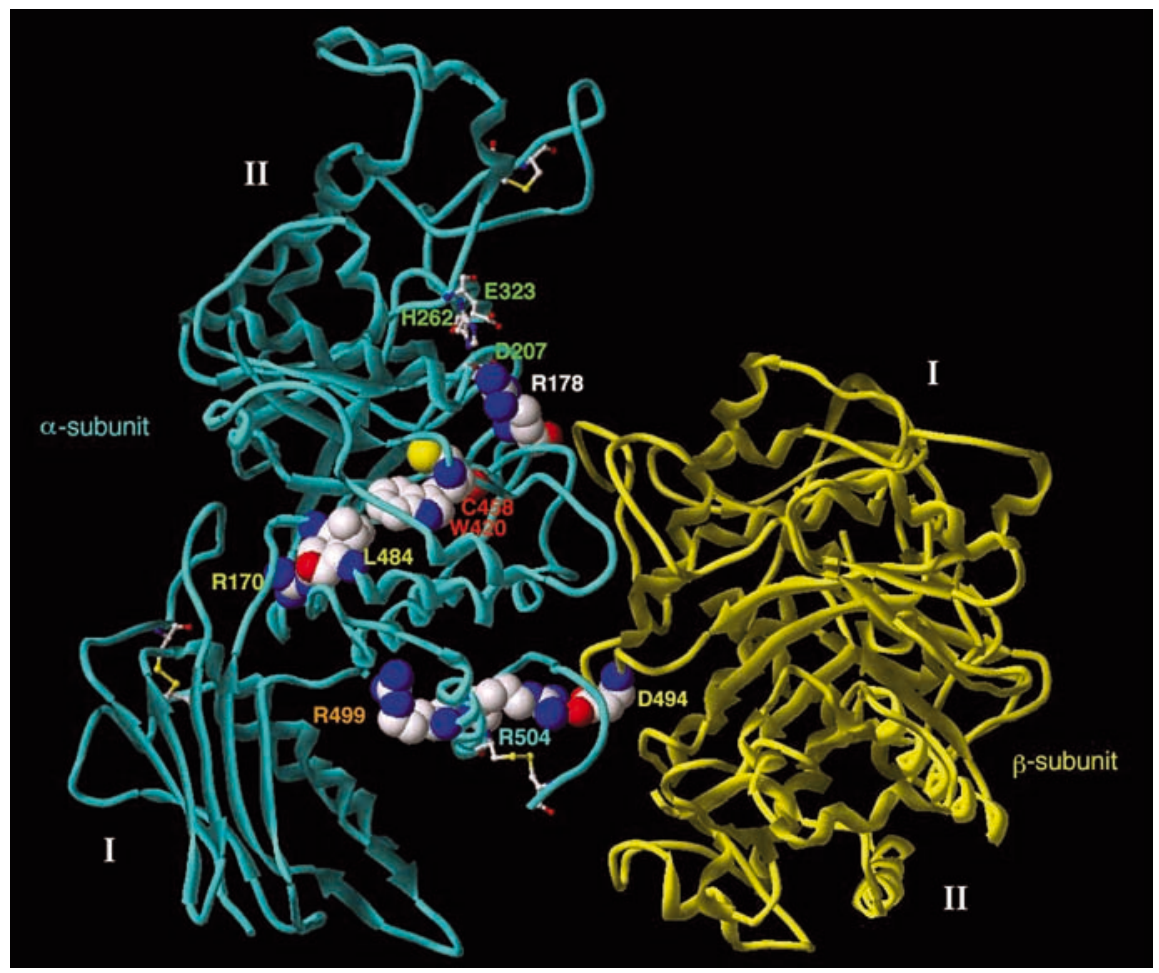

Group 4 (R499C and R499H)

R499 is located in the extra-helix like R504; however, this residue points inside the molecule (Fig. 1). R499 forms hydrogen bonds with the side chain of E528 of the barrel and the main chain of L43 in domain I, which are observed in the $\beta$-subunit as well. Substitution of $\mathrm{R} 499 \mathrm{C} / \mathrm{H}$ caused a disruption of these hydrogen bonds and a moderate conformational change near the residue (Fig. 4b). 
Table 2 Locations of the amino acid substitutions in the $\beta$-hexosaminidase $\alpha$-subunit and the biochemical defects

\begin{tabular}{lll}
\hline Group & Mutation & Mutation location \\
\hline 1 & R178H & $\begin{array}{l}\text { The active site in the barrel } \\
\text { The opposite side of the active } \\
\text { site in the barrel }\end{array}$ \\
3 & R504C, R504H & $\begin{array}{l}\text { The outside of the extra-helix } \\
\text { in domain II } \\
\text { The inside of the extra-helix } \\
\text { in domain II } \\
\text { The } \beta \text {-strands of domain II } \\
\text { near domain I }\end{array}$ \\
5 & R499C, R499H & R170W, L484P
\end{tabular}

${ }^{\mathrm{a}}$ Kytzia et al. (1983);

${ }^{\mathrm{b}}$ Tanaka et al. (1990), Tanaka et al. (1994);

${ }^{c}$ Paw et al. (1990), Paw et al. (1991);

Biochemical defect

The mutant $\alpha$ had the B1-type catalytic defect ${ }^{\mathrm{a}}$

The expressed mutant $\alpha$ did not express any catalytic activity ${ }^{\mathrm{b}}$,

and the amount of the mature $\alpha$ was decreased in the patients' cells

The mutant $\alpha$ could not associate with $\beta^{\mathrm{c}}$

The expressed mutant $\alpha$ was retained in the endoplasmic reticulum ${ }^{\mathrm{d}}$

The expressed mutant $\alpha$ did not express catalytic activity ${ }^{\mathrm{e}}$,

and the amount of the mature $\alpha$ was decreased in the patients' cells

${ }^{\mathrm{d}}$ Paw et al. (1990), Mules (1992);

${ }^{\mathrm{e}}$ Tanaka et al. (1994)
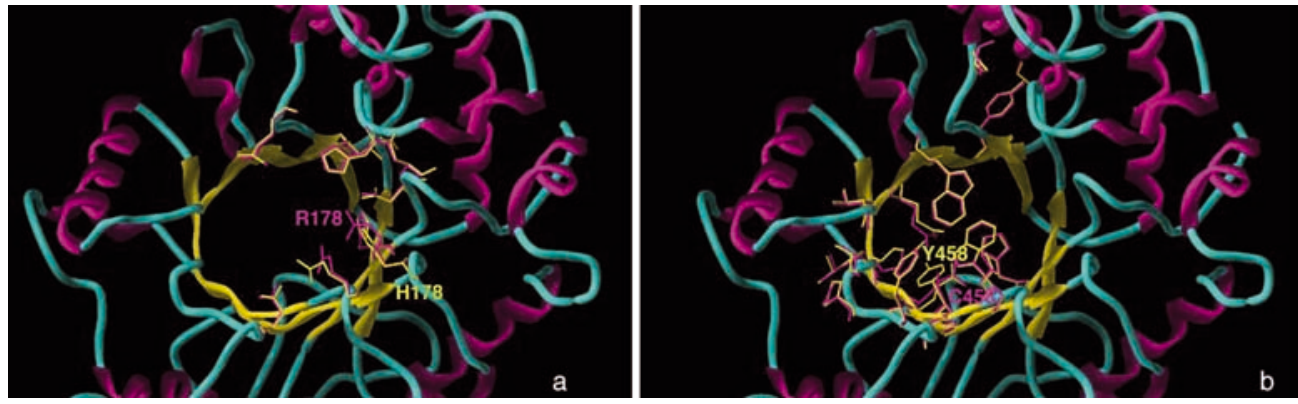

Fig. 3 Structural changes in the active site pocket of the Hex $\alpha$ subunit caused by $\mathrm{R} 178 \mathrm{H}$ and $\mathrm{C} 458 \mathrm{Y}$. The influence of substitution $\mathrm{R} 178 \mathrm{H}$ in group 1 and $\mathrm{C} 458 \mathrm{Y}$ in group 2 are shown in $\mathbf{a}$ and $\mathbf{b}$, respectively. Only the side chains of the residues influenced by each substitution are shown. The residues of the wild type and mutants are colored magenta and yellow, respectively

Fig. 4 Structural changes caused by R504C, R499C and R170 W. The influence of substitution R504H in group 3, R499H in group 4, and $\mathrm{R} 170 \mathrm{~W}$ in group 5 are shown in $\mathbf{a}, \mathbf{b}$, and $\mathbf{c}$, respectively. The ribbon model of domain I and domain II are colored white and blue, respectively. Only the side chains of the residues influenced by each substitution are shown. The residues of the wild type and mutants are colored magenta and yellow, respectively

Group 5 (R170W and L484P)

The R170 and L484 residues are located in the first $\beta$ strand and the eighth $\alpha$-helix, respectively, in domain II facing domain I, and these residues are adjacent to each other in the three-dimensional structure (Fig. 1). The side chain of R170 in domain II forms hydrogen bonds with E141 in domain I, which are observed in the $\beta$ subunit structure as well. These hydrogen bonds are thought to contribute to stabilization of domains I and II. Substitution of R170 to W results in disruption of these hydrogen bonds between the two domains. R170 also forms hydrogen bonds with the main chain $\mathrm{O}$ atoms of F167 and K197 in domain II, and these residues are
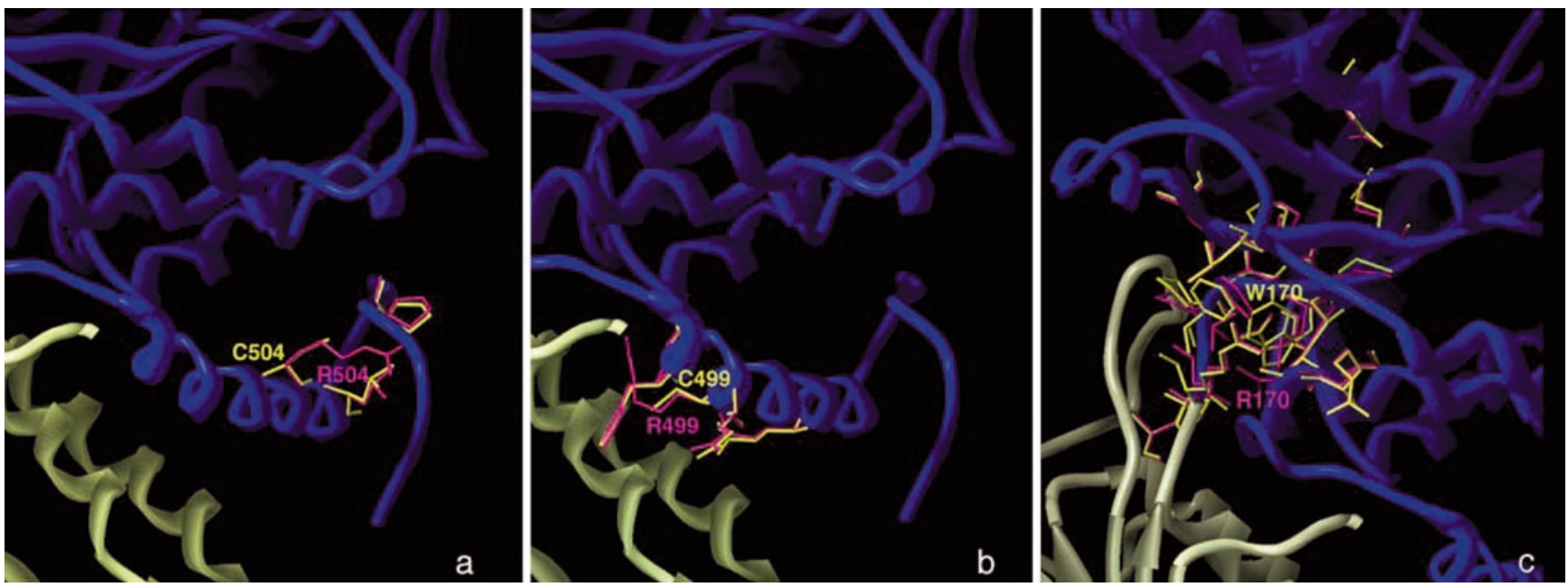
packed tightly. The substitution of R170 to W with a bulkier side chain has a large effect on the interface of domains I and II (Fig. 4c).

L484 is located on the eighth $\alpha$-helix, which is adjacent to domain I. Substitution of L484 to P caused a disruption of the hydrogen bond pattern inside the $\alpha$ helix and affected the residues of the $\alpha$-helix (data not shown).

\section{Discussion}

The catalytic domain structure of the human Hex $\alpha$ subunit had been modeled using the structure of chitobiose from S. marcescens (Tews et al. 1996). However, it was restricted to the predicted catalytic region because of the low-sequence identity with the human Hex $\alpha$ subunit. Recently, the crystal structure of the human Hex B was determined (Mark et al. 2003). This structural data allowed us to build the entire structure model of the human Hex $\alpha$-subunit and the heterodimer, HexA.

Based on the structural information, we tried to gain insight into the pathogenesis of the GM2 gangliosidosis $B$ variant resulting from specific amino acid substitutions. According to the structural defects, we divided the nine mutations into five groups. The substitution in group 1 is deduced to have a direct effect on the active site. In group 2, the structural changes were observed around the active site pocket, and these are likely to have a drastic destabilizing effect on the active site pocket. In group 3 , the substitutions are deduced to introduce a disruption of the binding with D494 in the $\beta$ subunit for dimerization. In group 4, the substitutions are deduced to decrease the stability around the extra-helix packed into domain I and the barrel. In group 5, the substitutions are deduced to cause a drastic structural change in the interface between domains I and II.

The $\mathrm{R} 178$ residue is located close to the active site and is thought to be involved in substrate binding. In the $\beta$-subunit complex with $\mathrm{N}$-acetylglucosamine-thiazoline (NGT), R211 corresponding to R178 in the $\alpha$-subunit is connected directly with both O-3 and O-4 of NGT through hydrogen bonds (Mark et al. 2003). R178H results in substitution of $\mathrm{H}$ for $\mathrm{R} 178$, which is an important residue for substrate binding. In addition, this substitution is deduced to cause a conformational change in residues that are responsible for the activity. This amino acid substitution is supposed to affect the enzymatic activity of Hex A directly and to increase the apparent $\mathrm{Km}$ value for a substrate. Similar structural changes were observed for R178C (Tanaka et al. 1990) and R178L (Triggs-Raine et al. 1991) (data not shown). The $\mathrm{R} 178 \mathrm{H}$ mutation has been identified in patients with the B1 variant of the disease, including case 1 (Tanaka et al. 1990; Ohno and Suzuki 1988; Kytzia et al. 1983; dos Santos et al. 1991; Tanaka et al. 1988).

The B1 variant of the disease is an unusual biochemical phenotype. Patients with this type express both
Hex A and Hex B activities, as assayed with 4-methylumbelliferyl GlcNAc or GalNAc, but the mutant Hex A is unable to hydrolyze 4-methylumbelliferyl GlcNAc-6-S (Grave et al. 2001; Kytzia et al. 1983). Brown et al. (1989) introduced the R178H mutation at the homologous site in the Hex $\beta$-subunit to examine the effect of the mutation on the $\beta$-subunit and showed biochemically that the resulting Hex B, expressed in COS cells, was catalytically inactive, although biosynthetic processing and stability were not affected. That is consistent with the result in which the conformational change by $\mathrm{R} 178 \mathrm{H}$ was only observed inside the active site pocket and does not have a significant influence.

The mutations in group 2 (W420C and $\mathrm{C} 458 \mathrm{Y}$ ) were found around the active site pocket (Fig. 1). In contrast with $\mathrm{R} 178 \mathrm{H}$, these substitutions are deduced to cause a drastic structural change widely around the active site pocket. Therefore, these substitutions are deduced to destabilize the barrel structure as well as the active site pocket rather than influence the active site directly. As shown in Fig. 3, the substitution of $\mathrm{C} 458$ to $\mathrm{Y}$ had a drastic effect on the active site pocket, including the residues forming the hydrophobic core (W373 and W460), which is responsible for stabilization of the barrel structure. The substitution of W420 to C with a smaller side chain is deduced to introduce a cavity into the hydrophobic packing in the barrel, as well as to cause disruption of the hydrophobic interactions in the interior of the barrel structure. Thereby, it would destabilize the structure near the active site pocket.

Tanaka et al. previously reported a patient with classical Tay-Sachs disease (Tanaka et al. 1990). The father and mother of the patient were of Irish and German descent respectively. The patient was a compound heterozygote with one allele carrying the W420C mutation, but that on the other allele was unidentified. Expression of the W420C mutation in COS 1 cells showed that it failed to express any catalytic activity with either a sulfated or nonsulfated substrate. Tanaka et al. suggested that W420C might cause new disulfide bonding. In the $\mathrm{W} 420 \mathrm{C}$ model, a free cysteine residue, $\mathrm{C} 458$ is found near $\mathrm{C} 420(\mathrm{C} \alpha-\mathrm{C} \alpha$ distance $=6.2 \AA)$, although their side chains are on opposite sides of the $\beta$ sheet. The formation of a disulfide bond between C420 and $\mathrm{C} 458$ could cause a much larger structural change. Expression analysis was also performed on the $\mathrm{C} 458 \mathrm{Y}$ mutation, and the mutant cDNA did not express any catalytic activity of Hex A (Tanaka et al. 1994). The results of Western blotting analysis of cell homogenates from cases 2 and 3 showed that the amount of mature $\alpha$ subunit was decreased in both cases (unpublished data).

The substitutions in group 3 result in a disruption of the interaction between the $\alpha$ - and $\beta$ - subunits in the $\alpha \beta$ heterodimer. In the $\beta \beta$ homodimer, R501, corresponding to R504 in the $\alpha$-subunit, binds directly with D494 at the dimer interface. R504 in the $\alpha$-subunit is deduced to be a partner of $\mathrm{D} 494$ in the $\beta$-subunit in the $\alpha \beta$ heterodimer, creating an essential bond at the dimer interface (Fig. 2). R504C/H introduced a disruption in this 
essential binding for dimerization. The substitution is deduced to cause a moderate conformational change in the dimer interface (Fig. 4a)

Paw et al. (1990) reported a patient with the juvenile type of the disease. The patient was homozygous for $\mathrm{R} 504 \mathrm{H}$, and cultured fibroblasts from the patient synthesized a precursor $\alpha$-subunit, but the mutant $\alpha$-subunit failed to associate with the $\beta$-subunit to form an active heterodimer. Furthermore, they reported that R504C gave rise to a mutant $\alpha$-subunit with the same biochemical defects as those in the case of $\mathrm{R} 504 \mathrm{H}$ (Paw et al. 1991). The expressed products were secreted as the $\alpha$-monomer rather than a dimer of the $\alpha$-subunits. These biochemical results are consistent with the deduced structural defect by $\mathrm{R} 504 \mathrm{C} / \mathrm{H}$. The substitution of the $\mathrm{R} 504$ - which is responsible for dimerization - by a C or an $\mathrm{H}$ residue, is deduced to affect dimerization. Cases 4 and 5 , having the $\mathrm{R} 504 \mathrm{C} / \mathrm{H}$ mutation on one allele and the splicing mutation (IVS5, $-1 \mathrm{G} \rightarrow \mathrm{T}$ ) on the other allele, exhibit the classical Tay-Sachs phenotype. The combination of the mutations may cause expression of low enzyme activity and clinically the severe form of the disease because the latter does not express any catalytic activity.

R499 and R504 are present on the same extra-helix, but the structural defects caused by these substitutions are completely different. The R499 residue was deduced to form hydrogen bonds with domain I and the barrel structure. R499 would be one of the residues for stabilizing the two domains. The substitution R499 to $\mathrm{C}$ or $\mathrm{H}$ results in disruption of these intramolecular hydrogen bonds. Thus, it is deduced to cause a loss of stability in this region but not to affect the active site. In cells from the patients with $\mathrm{R} 499 \mathrm{C} / \mathrm{H}$ described here, residual Hex A activity was detected, and they clinically expressed the juvenile type of the disease. Paw et al. (1990) described a patient with the juvenile type of the disease carrying the R499H mutation on one allele and 1278insTATC, which is a common Jewish Tay-Sachs mutation, on the other allele. Cultured fibroblasts from the patient synthesized an $\alpha$-subunit that was retained in the endoplasmic reticulum. The abnormal $\alpha$-subunit did not acquire the mannose 6-phosphate marker, nor was it secreted from the cells in the presence of $\mathrm{NH}_{4} \mathrm{Cl}$. The $\mathrm{R} 499 \mathrm{C}$ mutation was previously found in a patient with the adult type of the disease by Mules et al. (1992). The patient was of mixed origin, including Slavic, Irish, English, and Polish.

The mutations in group 5 (R170W and L484P) are in domain II facing domain I (Fig. 1). These substitutions are deduced to affect the interface between domains I and II. The R170 residue forms hydrogen bonds with E141 in domain I. In the $\beta$-subunit, the corresponding $\mathrm{R}$ and $\mathrm{E}$ residues are also conserved, and these residues form hydrogen bonds directly. These hydrogen bonds are thought to contribute to stabilization of domains I and II. Through these substitutions, these hydrogen bonds would be disrupted, and the surrounding residues would be affected. R170W is likely to have a significant destabilizing effect on the domain interface (Fig. 4c); thus, it would cause degradation of the $\alpha$-subunit. The R170W mutation was also detected in Tay-Sachs patients of French Canadian origin (Fernandes et al. 1992).

As for the L484P mutation, an expression study was performed, but the mutant cDNA did not produce any Hex A activity (Tanaka et al. 1994). The structural change caused by L484P was smaller than that by R170W (data not shown), but introducing P into the $\alpha$ helix is deduced to destabilize the structure of the $\alpha$-helix adjacent to domain I. Western blotting analysis revealed that the amount of the mature $\alpha$-subunit was decreased in cells from cases 8 and 9 carrying these mutations (unpublished data).

In conclusion, we determined the structural basis of the GM2 gangliosidosis B variant. The data indicate that heterogeneous structural changes in the Hex $\alpha$ subunit occur in this disease, and the results can well explain the pathogenesis. Further comparative studies from structural and biochemical aspects will clarify the molecular pathology of the disease. Structural study is useful for clarifying the pathogenesis of the disease.

Acknowledgements This work was partly supported by grants from the Tokyo Metropolitan Government; The Japan Society for the Promotion of Science; and the Ministry of Health, Labor and Welfare of Japan.

\section{References}

Akli S, Chelly J, Lacorte J-M, Poenaru L, Kahn A (1991) Seven novel Tay-Sachs mutations detected by chemical mismatch cleavage of PCR-amplified cDNA fragments. Genomics $11: 124-134$

Akli S, Chomel J-C, Lacorte J-M, Bachner L, Poenaru A, Poenaru L (1993) Ten novel mutations in the HEXA gene in non-Jewish Tay-Sachs patients. Hum Mol Genet 2:61-67

Berman HM, Westbrook J, Feng Z, Gilliland G, Bhat TN, Weissig H, Shindyalov IN, Bourne PE (2000) The Protein Data Bank. Nucleic Acids Research 28:235-242

Boustany R-MN, Tanaka A, Nishimoto J, Suzuki K (1991) Genetic cause of a juvenile form of Tay-Sachs disease in a Lebanese child. Ann Neurol 29:104-107

Brown CA, Neote K, Leung A, Gravel RA, Mahuran DJ (1989) Introduction of the $\alpha$ subunit mutation associated with the B1 variant of Tay-Sachs disease into the $\beta$ subunit produces a $\beta$ hexosaminidase $\mathrm{B}$ without catalytic activity. J Biol Chem 264:21706-21710

Claessens M, van Cutsem E, Lasters I, Wodak S (1989) Modeling the polypeptide backbone with 'spare parts' from known protein structures. Protein Engng 2:335-345

dos Santos MR, Tanaka A, sá Miranda C, Ribero MG, Maia M, Suzuki K (1991) GM2-gangliosidosis B1 variant: Analysis of $\beta$ hexosaminidase $\alpha$ gene mutations in 11 patients from a defined region in Portugal. Am J Hum Genet 49:886-890

Fernandes M, Karplan F, Natowicz M, Prence E, Kolodny E, Kaback M, Hechtman P (1992) A new Tay-Sachs disease B1 allele in exon 7 in two compound heterozygotes each with a second novel mutation. Hum Mol Genet 1:759-761

Gravel RA, Kaback MM, Proia RL, Sandhoff K, Suzuki K, Suzuki K (2001) The GM2 gangliosidoses. In: Scriver CR, Beaudet AL, Sly WS, Valle D (eds) The metabolic and molecular bases of inherited disease, 8th edn. McGraw-Hill, New York, pp 3827 3876 
Jones TA, Thirup S (1986) Using known substructures in protein model building and crystallography. EMBO J 5:819-822

Kabsh W (1976) A solution for the best rotation to relate two sets of vectors. Acta Crystallogr A32:827-828

Kabsh W (1978) A discussion of the solution for the best rotation to relate two sets of vectors. Acta Crystallogr A34:922-923

Kytzia H-J, Hinrichs U, Maire I, Suzuki K, Sandhoff K (1983) Variant of GM2-gangliosidosis with hexosaminidase A having a severely changed substrate specificity. EMBO J 2:1201-1205

Maier T, Strater N, Schuette CG, Klingenstein R, Sandhoff K, Saenger W (2003) The X-ray crystal structure of human betahexosaminidase B provides new insights into Sandhoff disease. J Mol Biol 328: 669-681

Mark BL, Vocadlo DJ, Knapp S, Triggs-Raine BL, Withers SG, James MNG (2001) Crystallographic evidence for substrateassisted catalysis in a bacterial $\beta$-hexosaminidase. $\mathbf{J}$ Biol Chem 276:10330-10337

Mark BL, Mahuran DJ, Cherney MM, Zhao D, Knapp S, James MNG (2003) Crystal structure of human $\beta$-hexosaminidase B: Understanding the molecular basis of Sandhoff and Tay-Sachs disease. J Biol Chem 327:1093-1109

Mules EH, Hayflick S, Miller CS, Reynolds LW, Thomas GH (1992) Six novel deleterious and three neutral mutations in the gene encoding the $\alpha$-subunit of hexosaminidase A in non-Jewish individuals. Am J Hum Genet 50:834-841

Myerowitz R, Costigan FC (1988) The major defect in Ashkenazi Jews with Tay-Sachs disease is an insertion in the gene for the $\alpha$ chain of $\beta$-hexosaminidase. J Biol Chem 263:18587-18589

Nishimoto J, Tanaka A, Nanba A, Suzuki K (1991) Expression of the $\beta$-hexosaminidase $\alpha$ subunit gene with the four-base insertion of infantile Jewish Tay-Sachs disease. J Boil Chem 266:14306-14309

Ohno K, Suzuki K (1988) Mutation in GM2-gangliosidosis B1 variant. J Neurochem 50:316-318

Paw BH, Neufeld EF (1988) Normal transcription of the $\beta$-hexosaminidase $\alpha$-chain gene in the Ashkenazi Tay-Sachs mutation. J Hum Genet 263:3012-3015

Paw BH, Moskowitz SM, Uhrhammer N, Wright N, Kaback MM, Neufeld EF (1990) Juvenile GM2 gangliosidosis caused by substitution of histidine for arginine at position 499 or 504 of the $\alpha$-subunit of $\beta$-hexosaminidase. J Biol Chem 265:94529457

Paw BH, Wood LC, Neufeld EF (1991) A third mutation at the $\mathrm{CpG}$ dinucleotide of codon 504 and a silent mutation at codon 506 of the HEXA gene. Am J Hum Genet 48:1139-1146
Sakuraba H, Matsuzawa F, Aikawa S, Doi H, Kotani M, Lin H, Ohno K, Tanaka A, Yamada H, Uyama E (2002) Molecular and structural studies of the GM2 gangliosidosis 0 variant. $\mathbf{J}$ Hum Genet 47:176-183

Sutcliffe MJ, Hayes FR, Blundell TL (1987) Knowledge based modeling of homologous proteins, part II: rules for the conformations of substituted sidechains. Protein Engng 1:385-392

Suzuki K (1987) Enzymatic diagnosis of sphingolipidoses. Methods Enzymol. 138:727-762

Tanaka A, Ohno K, Suzuki K (1988) GM2-gangliosidosis B1 variant: A wide geographic and ethnic distribution of the specific $\beta$-hexosaminidase $\alpha$ chain mutation originally identified in a Puerto Rican patient. Biochem Biophys Res Commun 156:1015-1019

Tanaka A, Pennett HH, Suzuki K (1990) A new point mutation in the $\beta$-hexosaminidase $\alpha$-subunit gene responsible for infantile Tay-Sachs disease in a non-Jewish Caucasian patient (a Kpn mutant). Am J Hum Genet 47:567-574

Tanaka A, Ohno K, Sandhoff K, Maire I, Kolodny EH, Brown A, Suzuki K (1990) GM2-gangliosidosis B1 variant: Analysis of $\beta$ hexosaminidase $\alpha$ gene abnormalities in seven patients. Am J Hum Genet 46:329-339

Tanaka A, Sakuraba H, Isshiki G, Suzuki K (1993) The major mutation among Japanese patients with infantile Tay-Sachs disease: A g-to-t transversion at the acceptor site of intron 5 of the $\beta$-hexosaminidase $\alpha$ gene. Biochem Biophys Res Commun 192:539-546

Tanaka A, Sakazaki H, Murakami H, Isshiki G, Suzuki K (1994) Molecular genetics of Tay-Sachs disease in Japan. J Inher Metab Dis 17:593-600

Tanaka A, Fujimaru M, Choeh K, Isshiki G (1999) Novel mutations, including the second most common in Japan, in the $\beta$ hexosaminidase $\alpha$ subunit gene, and a simple screening of Japanese patients with Tay-Sachs disease. J Hum Genet 44:9195

Tews I, Perrakis A, Oppenheim A, Dautoer Z, Wilson KS, Vorgias CE (1996) Bacterial chitobiose structure provides insight into catalytic mechanism and the basis of Tay-Sachs disease. Nat Struct Biol 3:638-648

Triggs-Raine BL, Akerman BR, Clarke JT, Gravel RA (1991) Sequence of DNA flanking the exons of the HEXA gene, and identification of mutations in Tay-Sachs disease. Am J Hum Genet 49:1041-1054 